\title{
Detection of Ventricular Premature Beats Based on the Pressure Signals of a Hemodialysis Machine
}

\author{
Mattias Holmer (corresponding author) ${ }^{\mathrm{a}, \mathrm{c}}$, Juan Pablo Martnez ${ }^{\mathrm{b}}$, Eduardo \\ Gil $^{\mathrm{b}}$, Frida Sandberg ${ }^{\mathrm{a}}$, Bo Olde ${ }^{\mathrm{c}}$, Leif Sörnmo ${ }^{\mathrm{a}}$ \\ ${ }^{a}$ Department of Biomedical Engineering and Center for Integrative Electrocardiology, Lund \\ University,Box 118,Lund,Sweden.Email: mattias.holmer@bme.lth.se.com, \\ frida.sandberg@bme.lth.se, leif.sornmo@bme.lth.se \\ ${ }^{b}$ CIBER de Bioingeniera, Biomateriales y Nanomedicina (CIBER-BBN), Spain, and \\ Aragón Institute of Engineering Research (I3A), IIS-Aragón, University of Zaragoza, Spain. \\ Email: jpmart@unizar.es, edugilh@unizar.es \\ ${ }^{c}$ Baxter International Inc., Lund, Sweden. Email: bo_olde@baxter.com
}

\begin{abstract}
Monitoring of ventricular premature beats (VPBs), being abundant in hemodialysis patients, can provide information on cardiovascular instability and electrolyte imbalance. In this paper, we describe a method for VPB detection which explores the signals acquired from the arterial and the venous pressure sensors, located in the extracorporeal blood circuit of a hemodialysis machine. The pressure signals are mainly composed of a pump component and a cardiac component. The cardiac component, severely overshadowed by the pump component, is estimated from the pressure signals using an earlier described iterative method. A set of simple features is extracted, and linear discriminant analysis is performed to classify beats as either normal or ventricular premature. Performance is evaluated on signals from nine hemodialysis treatments, using leave-one-out crossvalidation. The simultaneously recorded and annotated photoplethysmographic signal serves as the reference signal, with a total of 149686 normal beats and 3574 VPBs. The results show that VPBs can be reliably detected, quantified by a Youden's $J$ statistic of 0.9 , for average cardiac pulse pressures exceeding $1 \mathrm{mmHg}$; for lower pressures, the $J$ statistic drops to 0.55 . It is concluded that the cardiac pressure signal is suitable for VPB detection, provided that the average cardiac pulse pressure exceeds $1 \mathrm{mmHg}$.
\end{abstract}


Keywords: Ventricular premature beats, extracorporeal pressure sensors, cardiac pressure signal, fistula, hemodialysis, linear discriminant analysis

\section{Highlights}

- A novel method for VPB detection is proposed, based on the signals from the arterial and the venous pressure sensors of a hemodialysis machine.

- Features describing amplitude, duration, and area, combined with linear discriminant analysis, is used for classification of normal beats and VPBs.

- It is shown that the cardiac pressure signal is suitable for VPB detection, provided that the average cardiac pulse pressure exceeds $1 \mathrm{mmHg}$. 


\section{Introduction}

It is well-known that ventricular premature beats (VPBs) are frequent in

10 moved [3]. Ventricular arrhythmias in dialysis patients have been studied in long-term, ambulatory electrocardiogram (ECG) recordings, showing that VPBs are much more frequent during hemodialysis than during the postdialysis period [4. Patients with regional wall motion abnormalities, ischemic heart disfistula, where the waves enter the extracorporeal blood circuit of the dialysis machine. In this blood circuit, the waves are measured by the arterial and the venous pressure sensors. A peristaltic blood pump generates a pulsatile blood flow through the extracorporeal circuit. The blood flows from an arterial needle back to the fistula through a venous needle. The amplitude of the pressure pulses generated by the blood pump is drastically larger than is the amplitude of the pressure pulses generated by the heart.

We have previously shown that a cardiac pressure signal can be extracted so from the signals produced by the arterial and the venous pressure sensors [6, see also [7. In these studies, we compared heart rate and heartbeat occurrence time estimated from the extracted cardiac pressure signal to the corresponding quantities obtained from the photoplethysmographic (PPG) signal. The results showed that the proposed method offers excellent accuracy of heart rate and

In the present paper, we investigate, for the first time, whether the extracted cardiac pressure signal is suitable for VPB detection. A set of features, describ- 
ing amplitudes, durations, and areas, is proposed and used to classify detected beats as either VPBs or normal based on linear discriminant analysis. Using leave-one-out crossvalidation, the performance is evaluated by comparing the results from the proposed classifier to the annotated reference PPG signals.

\section{Background}

\subsection{Cardiac Pressure Signal Estimation}

The cardiac pressure signal is estimated using the iterative method described

45 in [6]. The method alternates between modeling of separate arterial and venous pump components, and estimation of a cardiac pressure signal. The resulting estimate is based on both the arterial and the venous pressure signals, by mixing the arterial cardiac component with the venous cardiac component. The mixing consists of time shifting and averaging, where the time shift is determined by maximizing the correlation between the arterial and venous cardiac components. The arterial and the venous cardiac components are obtained by subtracting the arterial and the venous model pump components from the respective arterial and venous pressure signals. The arterial and the venous pump signal estimates are determined by subtracting the cardiac pressure signal estimate from the respective signals. The pump signal estimates are, in turn, used to iteratively refine the arterial and the venous model pump components, so that the pump component remainders in the cardiac pressure signal estimate are reduced. The iteration continues until the difference in successive cardiac pressure signal estimates no longer improves. The main building blocks of the method are shown in Fig. 1(a), where the input signals and the output signal are illustrated in Figs. 11(b) and (c), respectively.

\section{Experiment and Database}

\subsection{Clinical Study}

The data originate from a clinical study performed at Skåne University Hos65 pital, Lund, Sweden. The study was approved by the local ethical review board, 

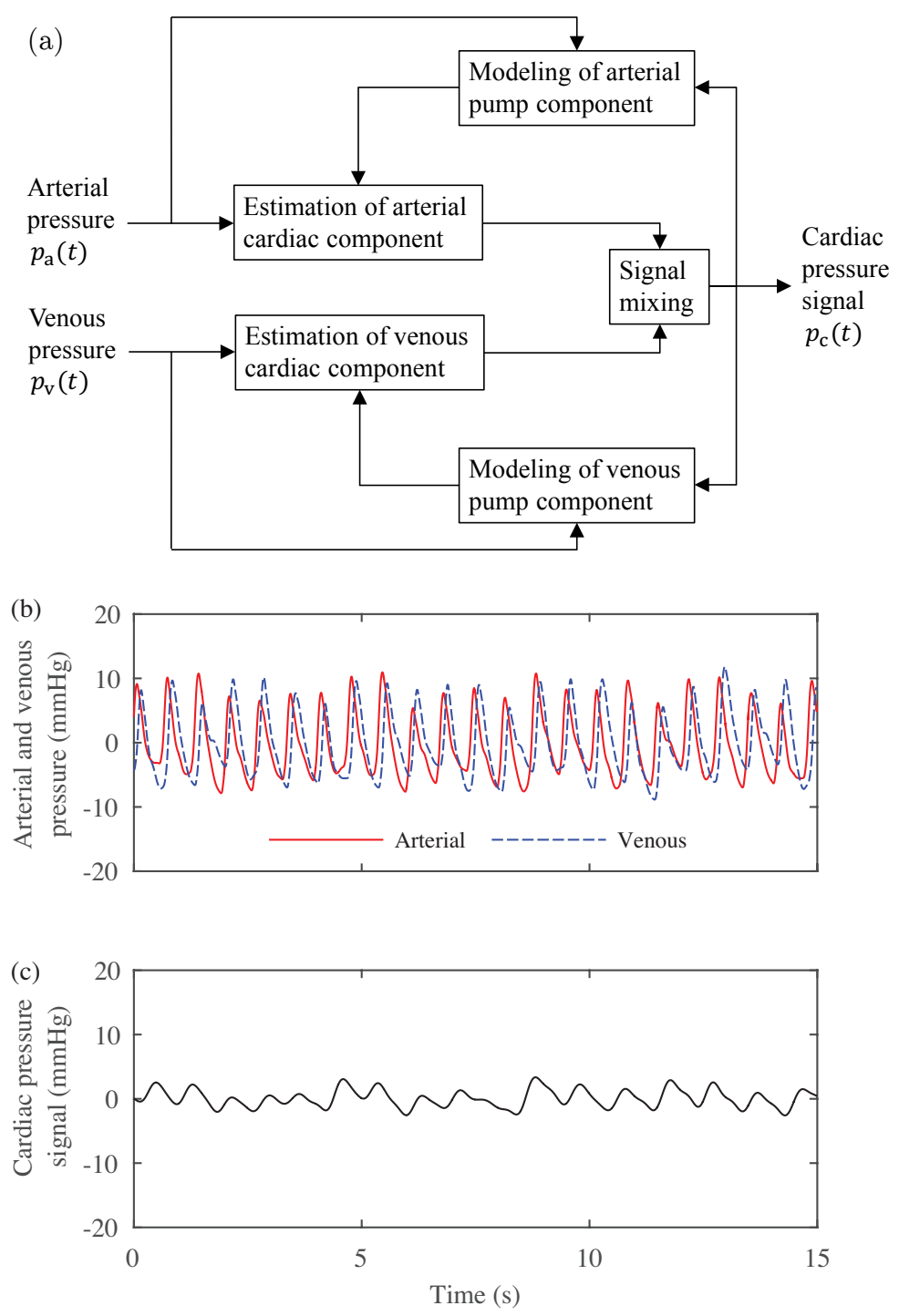

Figure 1: (a) Block diagram of the method for cardiac pressure signal estimation. Note that the output signal is referred to as "cardiac pressure signal", whereas the intermediate signals are referred to as "components". (b) The arterial and the venous pressure signals are the input to the method, whereas (c) the cardiac pressure signal is the output. Note the considerable difference in amplitude between the signals in (b) and (c). 
and all patients signed an informed consent form before participating.

The data set includes 9 treatments from 7 patients with kidney failure who underwent hemodialysis treatment for at least three months prior to the study onset. The treatments were performed according to the regular prescription provided by the nephrologist, and lasted 4 to 5 hours. Four patients had a history of heart complication. One patient had a graft as vascular access, whereas all others had fistulas. The average cardiac pulse pressure $\bar{P}_{\mathrm{np}}$ was determined during a blood pump stop at the treatment onset, see Table1. Treatments with $\bar{P}_{\text {np }}$ below $0.5 \mathrm{mmHg}$ [6], as well as patients with pacemaker, patients undergoing hemodiafiltration treatment, and patients participating in other studies, were not included.

The patients were treated with AK 200 hemodialysis machines from Gambro. An external device with pressure sensors was connected to the extracorporeal blood circuit to acquire the arterial and the venous pressure signals. The external device was used instead of the built-in pressure sensors to avoid the time-consuming work that comes with a software update of a dialysis machine. However, the recorded data can be regarded as originating from the built-in sensors, since the external and built-in sensors were of identical brand and type.

As reference, a PPG signal was acquired using the LifeSense ${ }^{\mathrm{TM}}$ finger pulse 85 oximeter. The PPG signal and the estimated cardiac pressure signal were lowpass filtered, using a cut-off frequency of $5 \mathrm{~Hz}$. All analyzed signals had a time resolution of $10 \mathrm{~ms}$. The use of the PPG signal as a reference is discussed in Section 6 .

\subsection{Annotation of the Reference PPG Signal}

Firstly, pulse detection was performed on the reference PPG signal, using a lowpass differentiator filter and a time-varying threshold, where the time of the peak amplitude of each heart pulse was used as reference [8, 9. Secondly, the detected pulses were classified as either normal or VPB using the method in [9. Next, all VPBs were manually reviewed to avoid incorrect annotations. 95 Segments with motion artifacts were manually excluded, leading to that $9 \%$ of 
Table 1: Treatment characteristics

\begin{tabular}{rrrrrrr}
\hline $\begin{array}{r}\text { Treatment } \\
\#\end{array}$ & $\begin{array}{r}\text { Duration } \\
(\mathrm{h}: \mathrm{mm})\end{array}$ & $\begin{array}{r}\text { Discarded } \\
(\mathrm{h}: \mathrm{mm})\end{array}$ & $\begin{array}{r}\bar{P}_{\mathrm{np}} \\
(\mathrm{mmHg})\end{array}$ & $\begin{array}{r}m_{\mathrm{pp}} \pm \sigma_{\mathrm{pp}} \\
(\mathrm{ms})\end{array}$ & \#VPBs & \#normal \\
\hline 1 & $4: 13$ & $0: 10$ & 5.0 & $993 \pm 109$ & 158 & 14526 \\
2 & $4: 10$ & $0: 03$ & 2.9 & $952 \pm 175$ & 507 & 15029 \\
3 & $4: 31$ & $0: 11$ & 1.8 & $767 \pm 51$ & 6 & 19768 \\
4 & $4: 41$ & $0: 04$ & 1.3 & $785 \pm 107$ & 336 & 20852 \\
5 & $4: 40$ & $0: 26$ & 1.3 & $966 \pm 111$ & 54 & 15665 \\
6 & $4: 54$ & $1: 49$ & 0.70 & $790 \pm 72$ & 23 & 13879 \\
7 & $4: 52$ & $0: 04$ & 0.70 & $756 \pm 134$ & 773 & 22060 \\
8 & $4: 37$ & $0: 02$ & 0.55 & $871 \pm 191$ & 1382 & 17586 \\
9 & $3: 42$ & $0: 51$ & 0.54 & $961 \pm 180$ & 335 & 10321 \\
\hline
\end{tabular}

The category "Discarded" is the accumulated duration of discarded segments due to motion artifacts in the PPG signal. $\bar{P}_{\mathrm{np}}$ is the average pulse pressure of the cardiac pressure signal determined during blood pump stop. $m_{\mathrm{pp}}$ and $\sigma_{\mathrm{pp}}$ denote the mean and standard deviation, respectively, of the peak-to-peak interval. Note that the number of VPBs and normal beats, $m_{\mathrm{pp}}$, and $\sigma_{\mathrm{pp}}$ are determined from the annotated PPG signals.

the total treatment time were excluded. For each treatment, Table 1 presents its duration, the duration of discarded segments, $\bar{P}_{\mathrm{np}}$, the mean and standard deviation of the peak-to-peak interval in the PPG signal, and the number of annotated normal and premature beats.

\section{Methods}

\subsection{Preprocessing}

The cardiac pressure signal $p_{\mathrm{c}}(t)$ is estimated from the observed arterial and venous pressure signals, denoted $p_{\mathrm{a}}(t)$ and $p_{\mathrm{v}}(t)$, respectively, using the iterative method briefly described in Section 2.1. Segments in $p_{\mathrm{c}}(t)$ with excessive noise, manifested as sudden changes in energy (at least a factor of 4), or a heart rate lower than 15 or higher than 180 beats per minute, are excluded. Figures 2(a) and (b) illustrates $p_{\mathrm{a}}(t), p_{\mathrm{v}}(t)$, and $p_{\mathrm{c}}(t)$, respectively. 
Since the amplitude of $p_{\mathrm{c}}(t)$ varies significantly during treatment, $p_{\mathrm{c}}(t)$ is detrended and normalized to produce amplitude-independent features, using a method based on empirical mode decomposition [10, 11. The local extrema are identified in $p_{\mathrm{c}}(t)$. If two local extrema with the same polarity are closer than $350 \mathrm{~ms}$ to each other, then only the extremum with the largest magnitude is kept. If the peak-to-peak interval $T_{\mathrm{pp}}$ is 1.8-2.2 times larger than the 5point sliding median of $T_{\mathrm{pp}}$, denoted $\tilde{T}_{\mathrm{pp}}$, the previously excluded extremum is recovered, provided that a pulse with the peak-to-peak amplitude larger than $0.4 \mathrm{mmHg}$ exists in the interval $[0.85,1.15] \cdot \tilde{T}_{\mathrm{pp}}$. The upper envelope $e_{\max }(t)$ is obtained by connecting the local maxima using cubic spline interpolation. The
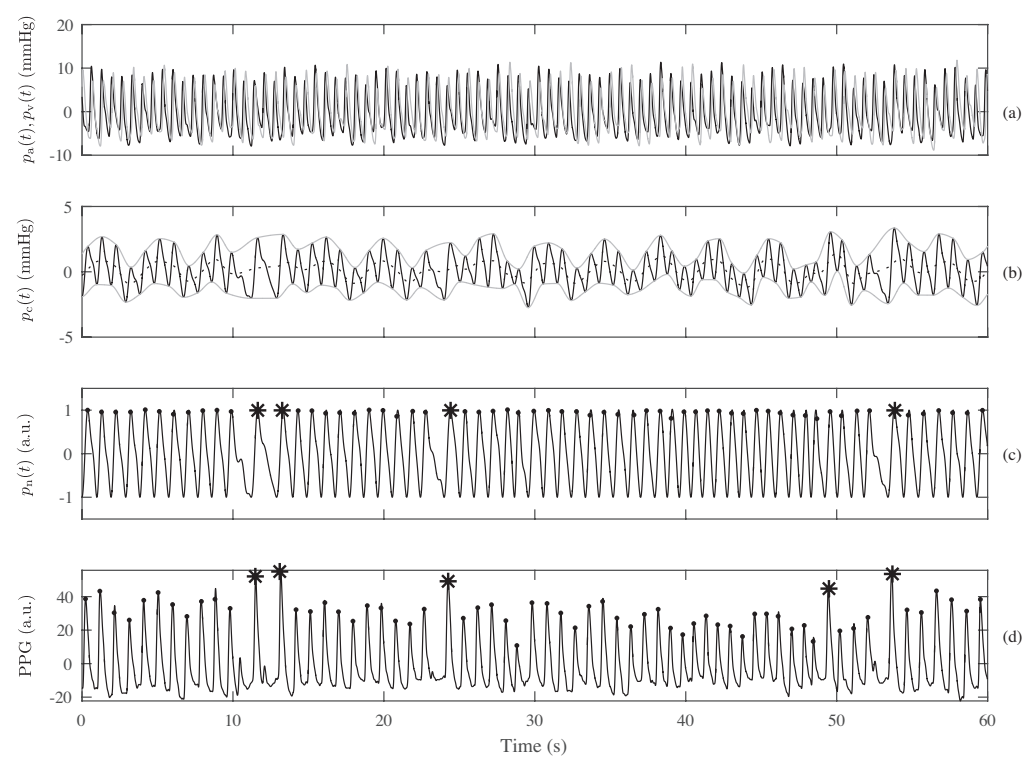

Figure 2: Illustration of signals and VPB detection for treatment \#1. (a) Venous pressure signal $p_{\mathrm{v}}(t)$ (black) and arterial pressure signal $p_{\mathrm{a}}(t)$ (grey). (b) Cardiac pressure signal $p_{\mathrm{c}}(t)$ estimated from $p_{\mathrm{v}}(t)$ and $p_{\mathrm{a}}(t)$ (black), the envelopes $e_{\max }(t)$ and $e_{\min }(t)$ (grey), and the trend $b(t)$ (dotted line). (c) The normalized cardiac pressure signal $p_{\mathrm{n}}(t)$; VPBs are labeled with "*” and normal beats with ".". (d) The reference PPG signal with annotations. Note the large difference in amplitude between $p_{\mathrm{c}}(t)$ in $(\mathrm{b})$ and $p_{\mathrm{v}}(t)$ and $p_{\mathrm{a}}(t)$ in (a). 
lower envelope $e_{\min }(t)$ is obtained in the same way, but instead connecting the local minima. The average envelope $\bar{e}(t)$ and the trend $b(t)$ are determined by

$$
\begin{aligned}
& \bar{e}(t)=\frac{1}{2}\left(e_{\max }(t)-e_{\min }(t)\right), \\
& b(t)=\frac{1}{2}\left(e_{\max }(t)+e_{\min }(t)\right),
\end{aligned}
$$

respectively. Figure 2(b) illustrates $e_{\max }(t), e_{\min }(t)$, and $b(t)$, together with $p_{\mathrm{c}}(t)$. The detrended and normalized cardiac pressure signal is obtained by

$$
p_{\mathrm{n}}(t)=\frac{p_{\mathrm{c}}(t)-b(t)}{\bar{e}(t)},
$$

illustrated in Fig. 2(c) along with information on beat type as produced by the method described below. Figure 2(d) shows the reference PPG signal and the annotations.

\subsection{Feature Extraction}

The simple features $X \in\{P, T, A\}$, illustrated in Fig. 3 and based on (a) amplitude $P$, (b) duration $T$, and (c) area $A$, are considered for classification. Four amplitude features are extracted from $p_{\mathrm{c}}(t)$, whereas 6 area features and 7 duration features are extracted from $p_{\mathrm{n}}(t)$ since this signal is much less influenced by variations in baseline and amplitude. Since a VPB may influence the features of the preceding beat $X^{-}$, as well as the following beat $X^{+}$, the features of the two enclosing beats are also evaluated, leading to an additional 34 features. Since the features may vary considerably within a treatment session, the median of the five most recent beats is used for centering, denoted with superscript ${ }^{\mathrm{c}}$, or normalizing the features, denoted with superscript ${ }^{\mathrm{n}}$, either with $X^{\mathrm{c}}=X-\tilde{X}$ or $X^{\mathrm{n}}=X / \tilde{X}$, thus leading to 102 additional features based on $X^{-}, X, X, X^{+}$. In addition, the difference between the current beat's peakto-peak interval and that of the preceding beat, denoted $\Delta T_{\mathrm{pp}}$, is included as a feature. In total 154 features are extracted, and considered for classification.

\subsection{Classification and Training}

Beats detected in $p_{\mathrm{c}}(t)$ are classified as either normal or ventricular premature, using linear discriminant analysis. For each beat, the following linear 

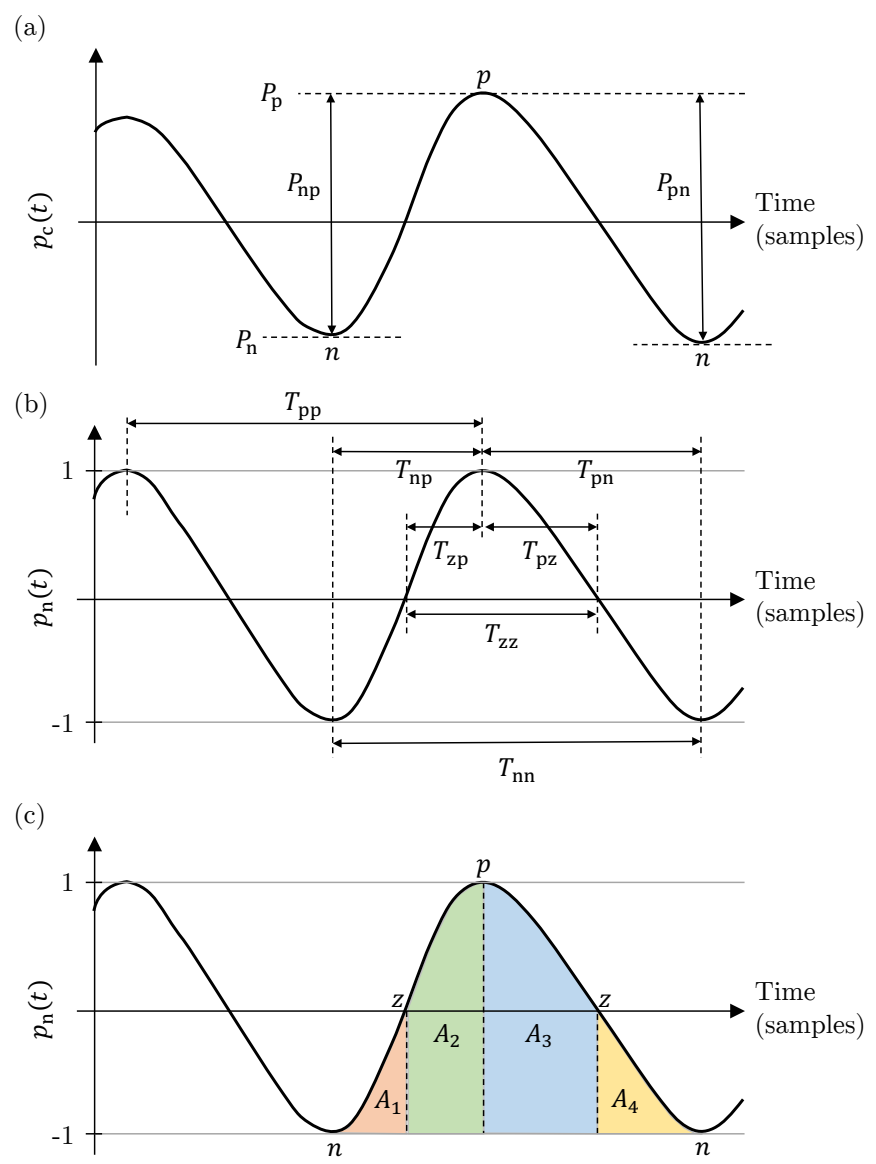

Figure 3: (a) Amplitude features determined from $p_{\mathrm{c}}(t)$. (b) Duration and (c) area features determined from $p_{\mathrm{n}}(t)$. The following area features are evaluated: $A=A_{1}+A_{2}+A_{3}+A_{4}$, $A_{\mathrm{pn}}=A_{3}+A_{4}, A_{\mathrm{np}}=A_{1}+A_{2}, A_{\mathrm{zz}}=A_{2}+A_{3}, A_{\mathrm{zp}}=A_{2}, A_{\mathrm{pz}}=A_{3}$. The vertical axis in (a) has arbitrary units.

discriminant function (LDF) is evaluated [12,

$$
\begin{aligned}
f(\mathbf{x})= & \left(\mathbf{m}_{\mathrm{v}}^{T}-\mathbf{m}_{\mathrm{n}}^{T}\right) \mathbf{C}^{-1} \mathbf{x} \\
& -\frac{1}{2} \mathbf{m}_{\mathrm{v}}^{T} \mathbf{C}^{-1} \mathbf{m}_{\mathrm{v}}+\frac{1}{2} \mathbf{m}_{\mathrm{n}}^{T} \mathbf{C}^{-1} \mathbf{m}_{\mathrm{n}}+\ln \left(\frac{P_{\mathrm{v}}}{P_{\mathrm{n}}}\right),
\end{aligned}
$$

where $\mathbf{x}$ is a vector containing the $K$ features of one beat, $\mathbf{m}_{\mathrm{v}}$ is the average feature vector for all VPBs, $\mathbf{m}_{\mathrm{n}}$ is the mean for all normal beats, $\mathbf{C}$ is the estimated covariance matrix of the features based on all beats. The a priori 

treatment determined from the annotations of all other treatments. A different LDF is used for each treatment, determined by leave-one-out cross validation, i.e., for each treatment the LDF is trained using all other treatments. If $f(\mathbf{x})>$ 0 , the beat is classified as VPB, otherwise as normal.

Youden's $J$ statistic [13] is used as performance index to determine the $K$ most relevant features,

$$
J=\frac{N_{\mathrm{TP}}}{N_{\mathrm{TP}}+N_{\mathrm{FN}}}+\frac{N_{\mathrm{TN}}}{N_{\mathrm{TN}}+N_{\mathrm{FP}}}-1,
$$

where $N_{\text {TP }}$ is the number of annotated VPBs classified as VPBs (true positives), $N_{\mathrm{FN}}$ is the number annotated VPBs classified as normal beats (false negatives), $N_{\mathrm{TN}}$ is the number of annotated normal beats classified as normal beats (true negatives), and $N_{\mathrm{FP}}$ is the number of annotated normal beats classified as VPBs (false positives). Note that perfect performance, i.e., $J=1$, is achieved for $N_{\mathrm{FN}}=0$ and $N_{\mathrm{FP}}=0$. The $J$ statistic was chosen as performance index since the data set is highly unbalanced, with many more normal beats than VPBs.

To determine the most relevant feature, LDFs with feature vector length of $K=1$ are first determined for all features separately. The feature whose LDFs (one for each treatment) yields the largest $J$ for the evaluated set of treatments, is judged as the most relevant. Then, the second most relevant feature is determined for LDFs when $\mathrm{x}$ contains two features, i.e., $K=2$. The feature which, together with the most relevant feature, results in the largest $J$ is judged as the second most relevant. The third most relevant feature is determined in the same way, and so on.

\section{Results}

Figure 4 presents the performance index $J$ as a function of the number of features. The performance index $J$ was evaluated for all 154 features, but only the 10 most relevant are plotted, since $J$ does not increase much when more than 5 features are included. For the complete data set the following five features were 


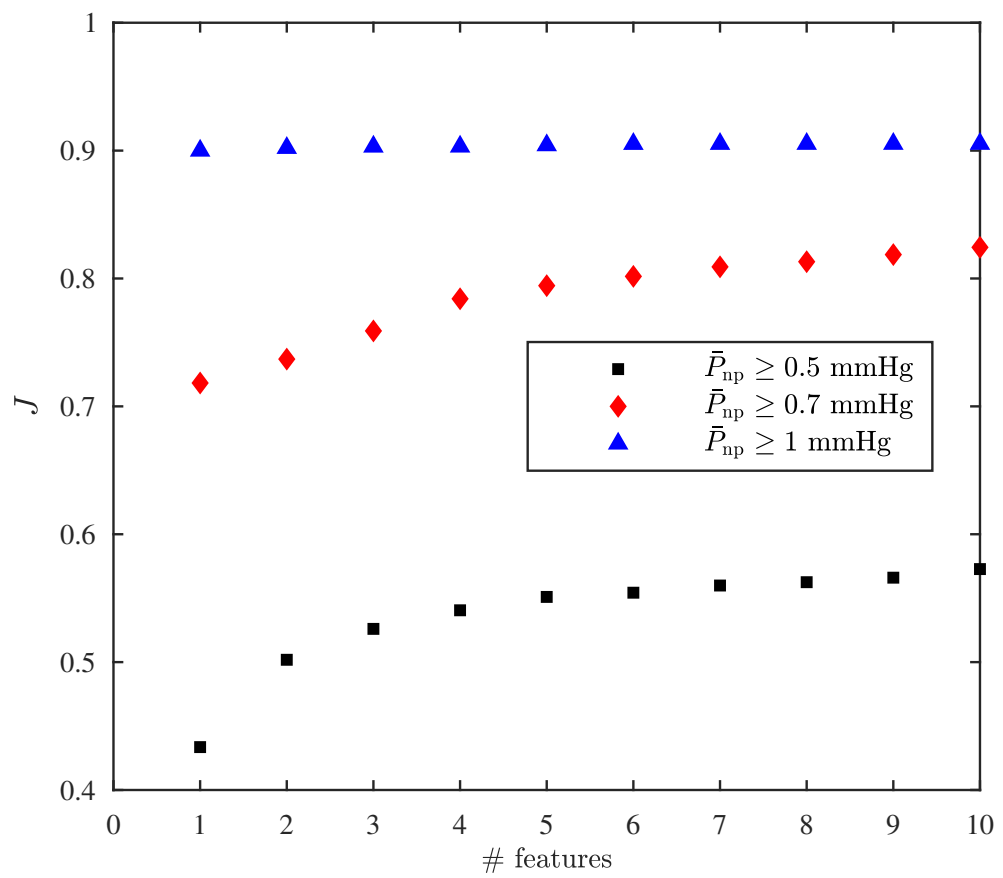

Figure 4: The performance index $J$ versus the number of features used for linear discriminant analysis when based on (black squares) all nine treatments, (red diamonds) seven treatments with $\bar{P}_{\mathrm{np}} \geq 0.7 \mathrm{mmHg}$, and (blue triangles) five treatments with $\bar{P}_{\mathrm{np}} \geq 1 \mathrm{mmHg}$.

found to be the most relevant $-T_{\mathrm{pp}}^{\mathrm{n}}, T_{\mathrm{zp}}^{\mathrm{c}}, T_{\mathrm{np}}^{\mathrm{c}-}, \Delta T_{\mathrm{pp}}$, and $P_{\mathrm{np}}^{\mathrm{c}-}$ - and therefore used for classification. When the data set is reduced to only include treatments with $\bar{P}_{\mathrm{np}} \geq 1 \mathrm{mmHg}, T_{\mathrm{pp}}^{n}$ remains the most relevant feature, whereas the order of relevance changes for the other features; the same observation applies to $\bar{P}_{\mathrm{np}} \geq 0.7 \mathrm{mmHg}$. From Fig. 4 it is noted that $J$ equals 0.9 when requiring that $\bar{P}_{\mathrm{np}} \geq 1 \mathrm{mmHg}$, while it drops to 0.55 when all treatments are analyzed.

Histograms of $f(\mathbf{x})$ for normal and VPBs are shown in Fig. 5 . Since the total number of normal beats is much larger than the total number of VPBs, the histograms have been normalized with respect to the total number of beats in each class. As expected, the two beat classes becomes more well-separated as the lower limit of $\bar{P}_{\mathrm{np}}$ increases.

The receiver operating characteristic (ROC) is shown in Fig. 6 when the 


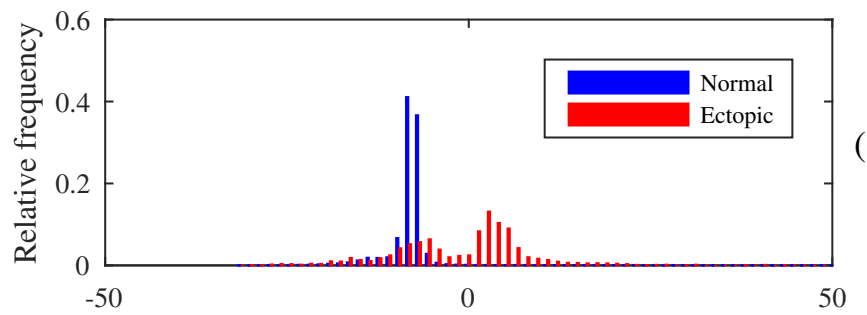

(a)
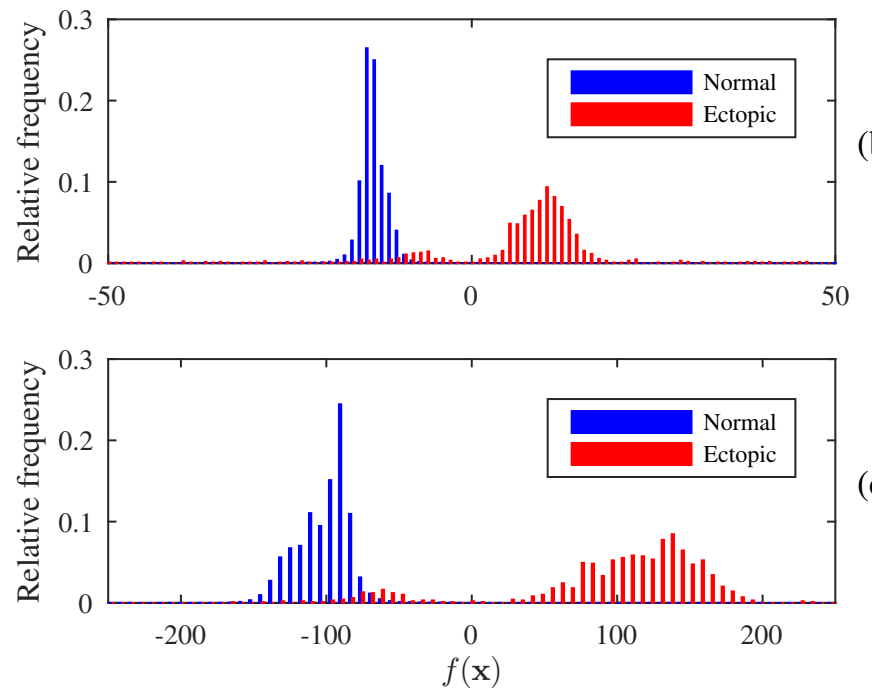

(c)

Figure 5: Normalized histograms of normal beats and VPBs as a function of the linear discriminant function $f(\mathbf{x})$, involving (a) all nine treatments, (b) seven treatments with $\bar{P}_{\mathrm{np}} \geq 0.7 \mathrm{mmHg}$, and (c) five treatments with $\bar{P}_{\mathrm{np}} \geq 1 \mathrm{mmHg}$.

five most significant features are used for classification. As expected, better performance is achieved as the lower limit of $\bar{P}_{\mathrm{np}}$ increases.

The ectopic beat count (EBC) is defined as the relative number of VPBs in a 10-min sliding window [5]. EBC is derived from both the estimated cardiac pressure signal and the annotations of the PPG signal, and shown for treatment \#1 in Fig. 7. The agreement between the EBC derived from the estimated cardiac pressure signal and the EBC derived from the annotations is satisfactory. The number of VPBs increases towards the end of the treatment - a behavior often observed in hemodialysis patients. The EBC for treatment \#2 fluctuates more over time, see Fig. 8 , but the agreement is still satisfactory. For both these 


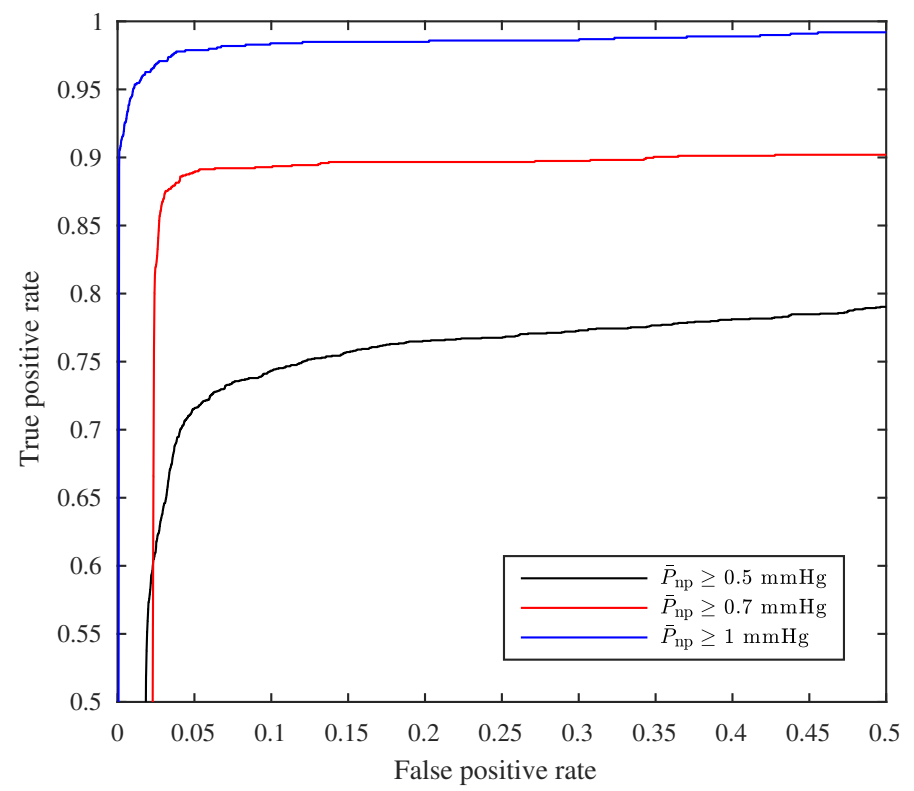

Figure 6: Receiver operating characteristic for VPB detection using linear discriminant analysis of the five most relevant features, for treatments with different lower limits of $\bar{P}_{\mathrm{np}}$.

treatments, $\bar{P}_{\text {np }}$ exceeds $1 \mathrm{mmHg}$. On the other hand, the agreement is much worse for treatment $\# 8$ due to that $\bar{P}_{\mathrm{np}}$ is very low $(0.55 \mathrm{mmHg})$, see Fig. 9 $\triangle \mathrm{EBC}$, i.e. the difference between the $\mathrm{EBC}$ derived from the pressure signal and the EBC derived from the annotations of the PPG signal, is computed, and its mean and standard deviation for all treatments is presented in Table 2 .

\section{Discussion}

We have demonstrated that the information provided by the extracorporeal arterial and venous pressure sensors can be used for detecting VPBs when the average cardiac pulse pressure $\bar{P}_{\mathrm{np}}$ exceeds $1 \mathrm{mmHg}$, previously suggested in $[6]$. When exceeding, the detection performance is similar to that achieved by analyzing the PPG. However, for $\bar{P}_{\mathrm{np}}<1 \mathrm{mmHg}$, the performance drops due to reduced separation between normal beats and VPBs, see Fig. 5 . Another manifestation of a low $\bar{P}_{\mathrm{np}}$ is that the number of VPBs is overestimated, see 


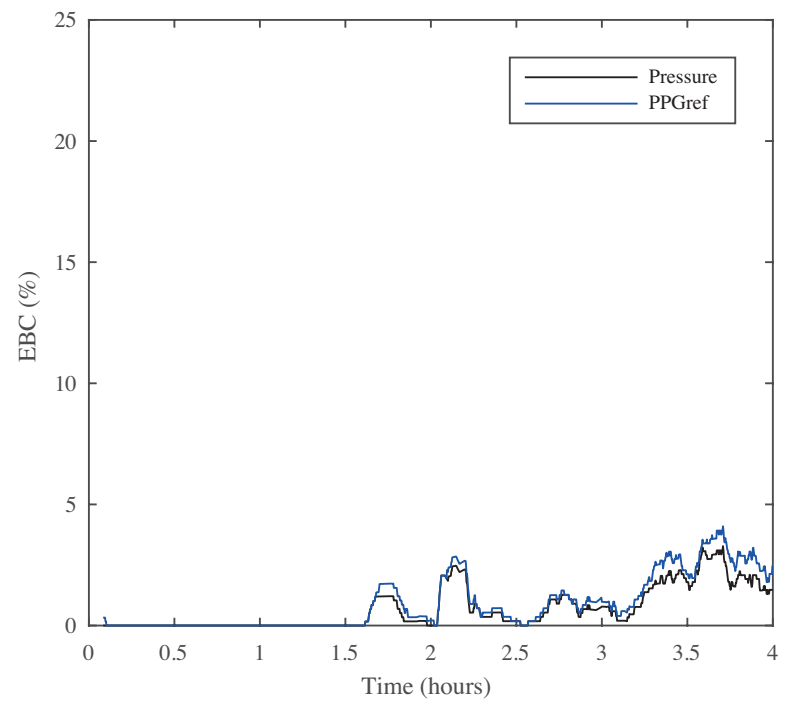

Figure 7: EBC versus treatment time, i.e. percentage of VPBs during a 10-min sliding window plotted versus time during treatment \#1. "PPGref" denotes the percentage of VPBs according to the annotations, and "Pressure" denotes the percentage of VPBs from detection.

Fig. 9, which is related to inaccurate estimation of the peak-to-peak interval $T_{\mathrm{pp}}$ in treatments with $\bar{P}_{\mathrm{np}}<1 \mathrm{mmHg}$. For the particular treatment in Fig. 9 the considerable variation in heart rate $\left(\sigma_{\mathrm{pp}}=191 \mathrm{~ms}\right.$, see Table 1 may have contributed to overestimation of VPBs.

In our experience, the properties of the fistula largely determines the amplitude of the cardiac pressure signal, and, accordingly, whether the patient is suitable for VPB detection. In addition, this amplitude may vary between different designs of hemodialysis machine. Unfortunately, the present data set is much too small to provide insight on the percentage of the hemodialysis population which is suitable for VPB detection with the present method.

For treatments with $\bar{P}_{\mathrm{np}} \geq 1 \mathrm{mmHg}$, the performance index $J$ does not improve when more than one feature are analyzed, see Fig. 4 . On the other hand, for $\bar{P}_{\mathrm{np}}<1 \mathrm{mmHg}, J$ improves with at least four additional features. Irrespective of $\bar{P}_{\mathrm{np}}, T_{\mathrm{pp}}^{\mathrm{n}}$ remains the most relevant feature on the present data 


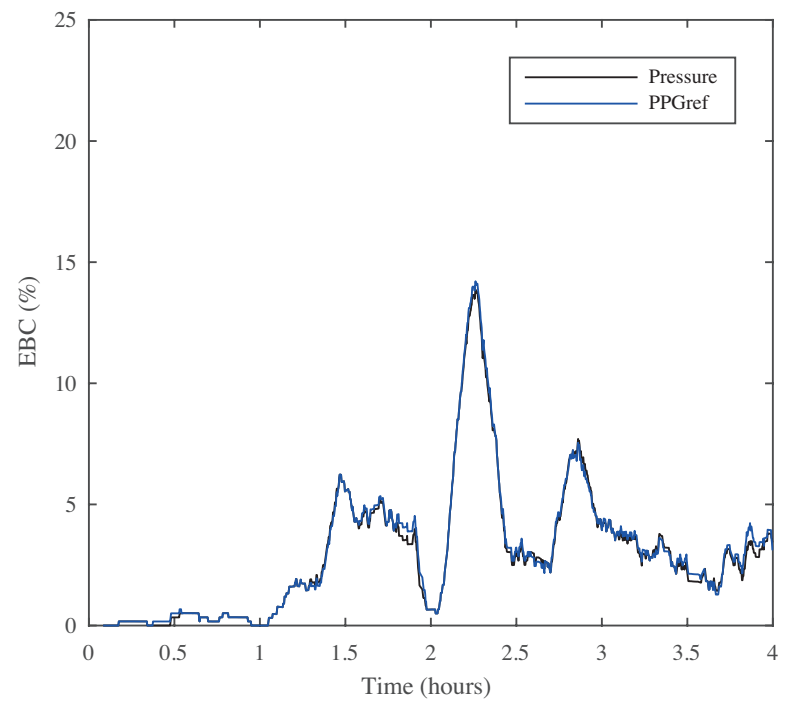

Figure 8: EBC versus treatment time, i.e. percentage of VPBs during a 10-min sliding window plotted versus time during treatment \#2. "PPGref" denotes the percentage of VPBs according to the annotations, and "Pressure" denotes the percentage of VPBs from detection.

set.

Given that the data set is unbalanced, the number of false positives will surpass the number of true positives for $\bar{P}_{\mathrm{np}}<1 \mathrm{mmHg}$, see Fig. 6. As a result, the classifier overestimates the number of VPBs for virtually any point on the ROC. On the other hand, for $\bar{P}_{\mathrm{np}} \geq 1 \mathrm{mmHg}$, a true positive rate of 0.9 is achieved with very few false positives.

Heartbeat classification is a well-studied problem in ECG signal processing, and many sophisticated techniques have emerged for this purpose, see, e.g., [14, [15, 16, 17. While those studies rest on solid knowledge on the properties of 215 the ECG signal, the present study explores heartbeat classification in relation to a novel type of signal whose properties have never before been explored for VPB detection. As a result, the present focus is more on feasibility than on methodological advances, and, therefore, well-known techniques are embraced such as empirical mode decomposition and linear discriminant analysis. 


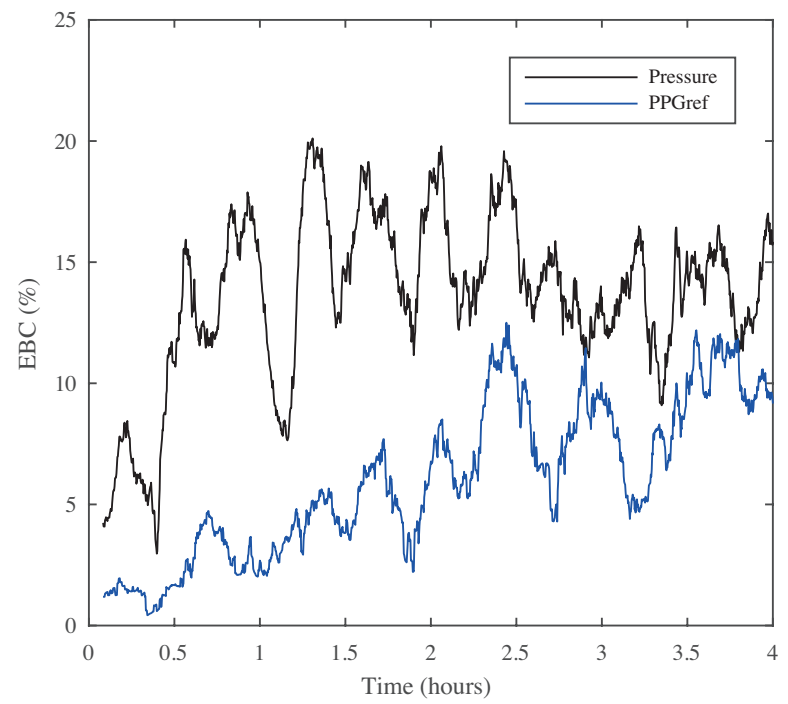

Figure 9: EBC versus treatment time, i.e. percentage of VPBs during a 10-min sliding window plotted versus time during treatment \#8. "PPGref" denotes the percentage of VPBs according to the annotations, and "Pressure" denotes the percentage of VPBs from detection.

A limitation of the present study is that the annotations of VPBs were not based on the ECG, but on the PPG.. When collecting the present database, it was unfortunately not practically feasible to record the ECG. However, we have previously shown that VPBs can be classified from the PPG signal, and that the PPG signal can be used as a surrogate for the ECG signal when analyzing heart rate turbulence [9, see also related work on automated VPB detection in the PPG signal [18, 19]. Although the ECG signal offers better temporal accuracy than does the PPG signal, we consider the accuracy of PPG-based classification to be sufficient when the goal is to evaluate detection performance for a method analyzing the cardiac pressure signal.

A major limitation of the present study is that the data set is much too small for a meaningful division into training and test sets. Therefore, the results of the present study should be viewed as a proof-of-concept that VPBs can be detected from the cardiac pressure signal. 
Table 2: Ectopic beat count

\begin{tabular}{rrrr}
\hline Treatment \# & $\bar{P}_{\mathrm{np}}$ & Mean $\Delta \mathrm{EBC}, \%$ & $\mathrm{Std} \Delta \mathrm{EBC}, \%$ \\
\hline 1 & 5.0 & -1.46 & 1.89 \\
2 & 2.9 & -0.40 & 1.18 \\
3 & 1.8 & 0.32 & 1.01 \\
4 & 1.3 & -0.52 & 2.41 \\
5 & 1.3 & 0.15 & 0.74 \\
6 & 0.70 & 0.49 & 2.39 \\
7 & 0.70 & 32.99 & 12.67 \\
8 & 0.55 & 23.63 & 11.91 \\
9 & 0.54 & -4.56 & 13.76 \\
\hline
\end{tabular}

$\bar{P}_{\mathrm{np}}$ is the average pulse pressure of the cardiac pressure signal determined during blood pump stop. $\triangle \mathrm{EBC}$ denotes the difference between classified and annotated EBC.

A limitation of the present method is its inability to distinguish between

235 also to the reference PPG method. In the ECG, atrial premature beats can be distinguished from ventricular premature beats since the QRS complex is preceded by an abnormal $\mathrm{P}$ wave. In the cardiac pressure signal, however, it is not possible to distinguish whether a heartbeat is preceded by an abnormal 240 P-wave.

\section{Conclusions}

The results show that VPB detection based on the cardiac pressure signal, estimated from the arterial and the venous pressure signals, is feasible. Satisfactory detection performance can be achieved when the average cardiac pulse 


\section{Acknowledgments}

The authors are grateful to Mårten Segelmark, Lena Mattsson, and Sarok Said at at Skåne University Hospital, Lund, Sweden, for carrying out the clinical investigation.

Bo Olde and Mattias Holmer are employed by Baxter International Inc. Bo Olde has a registered patent, WO2011080189(A1), related to the present paper.

This work was supported by the Swedish Research Council (00923/2011), the Crafoord Foundation, MINECO (Spain) through projects TIN2014-53567R and DPI2016-75458-R, and by European Social Fund and Aragón Government through consolidated group T96.

Ethical approval was given by the Regional Ethical Review Board in Lund, Dnr $314 / 2008$.

\section{References}

[1] O. M. Shapira, Y. Bar-Khayim, ECG changes and cardiac arrhythmias in chronic renal failure patients on hemodialysis, J. Electrocardiol. 25 (1992) 273-279.

[2] S. Abe, M. Yoshizawa, N. Nakanishi, T. Yazawa, K. Yokota, M. Honda, G. Sloman, Electrocardiographic abnormalities in patients receiving hemodialysis, Am. Heart J. 131 (1996) 1137-1144.

[3] A. Santoro, E. Mancini, R. Gaggi, S. Cavalcanti, S. Severi, L. Cagnoli, F. Badiali, B. Perrone, G. London, H. Fessy, L. Mercadal, F. Grandi, Electrophysiological response to dialysis: the role of dialysate potassium content and profiling, Contrib. Nephrol. 149 (2005) 295-305.

[4] J. O. Burton, S. Korsheed, B. J. Grundy, C. W. McIntyre, Hemodialysisinduced left ventricular dysfunction is associated with an increase in ventricular arrhythmia, Renal Failure 30 (2008) 701-709. 
[5] K. Solem, A. Nilsson, L. Sörnmo, An ECG-based method for early detection of abrupt changes in blood pressure during hemodialysis, ASAIO J. 52 (2006) 282-290.

[6] M. Holmer, F. Sandberg, K. Solem, B. Olde, L. Sörnmo, Cardiac signal estimation based on the arterial and venous pressure signals of a hemodialysis machine, Physiol. Meas. 37 (9) (2016) 1499-1515. doi: $10.1088 / 0967-3334 / 37 / 9 / 1499$.

[7] M. Holmer, F. Sandberg, K. Solem, E. Grigonyte, B. Olde, L. Sörnmo, Extracting a cardiac signal from the extracorporeal pressure sensors of a hemodialysis machine, IEEE Trans. Biomed. Eng. 62 (5) (2015) 1305-1315.

[8] J. Lázaro, E. Gil, J. M. Vergara, P. Laguna, Pulse rate variability analysis for discrimination of sleep-apnea-related decreases in the amplitude fluctuations of pulse photoplethysmographic signal in children, IEEE J. Biomed. Health Inform. 18 (2014) 240-246. doi:10.1109/JBHI. 2013.2267096.

[9] E. Gil, P. Laguna, J. P. Martínez, Ó. Barquero-Pérez, A. García-Alberola, L. Sörnmo, Heart rate turbulence analysis using photoplethysmography, IEEE Trans. Biomed. Eng. 60 (2013) 3149-3155.

[10] N. E. Huang, Z. Shen, S. R. Long, M. C. Wu, H. H. Shih, Q. Zheng, N.C. Yen, C. C. Tung, H. H. Liu, The empirical mode decomposition and the Hilbert spectrum for nonlinear and non-stationary time series analysis, Proc. Royal Soc. London A: Math.. Phys. Eng. Sci. 454 (1971) (1998) 903995. doi:10.1098/rspa.1998.0193

[11] G. Rilling, P. Flandrin, P. Gon, D. Lyon, On empirical mode decomposition and its algorithms, IEEE EURASIP Workshop on Nonlin. Signal and Image Process. 3 (2003) 8-11.

[12] R. O. Duda, P. E. Hart, D. G. Stork, Pattern Classification, 2nd Edition, Wiley-Interscience, New York, 2001. 
[13] W. J. Youden, Index for rating diagnostic tests, Cancer 3 (1) (1950) 32-

35. doi:10.1002/1097-0142(1950)3:1<32: :AID-CNCR2820030106>3.0. $\mathrm{CO} ; 2-3$.

[14] M. Lagerholm, C. Peterson, G. Braccini, L. Edenbrandt, L. Sörnmo, Clustering ECG complexes using Hermite functions and self-organizing maps, IEEE Trans. Biomed. Eng. 47 (2000) 838-848.

[15] I. Christov, G. Gómez-Herrero, V. Krasteva, I. Jekova, A. Gotchev, K. Egiazarian, Comparative study of morphological and time-frequency ECG descriptors for heartbeat classification, Med. Eng. \& Physics 28 (9) (2006) 876-887.

[16] I. Jekova, G. Bortolan, I. Christov, Assessment and comparison of different methods for heartbeat classification, Med. Eng. \& Physics 30 (2008) 248257.

[17] M. Llamedo, J. P. Martínez, An automatic patient-adapted ECG heartbeat classifier allowing expert assistance, IEEE Trans. Biomed. Eng. 59 (8) (2012) 2312-2320.

[18] T. Suzuki, K. I. Kameyama, T. Tamura, Development of the irregular pulse detection method in daily life using wearable photoplethysmographic sen-

口 sor, Int. Conf. IEEE EMBS (2009) 6080-6083doi:10.1109/IEMBS.2009. 5335401 .

[19] A. Sološenko, A. Petrènas, V. Marozas, Photoplethysmography-based method for automatic detection of premature ventricular contractions, IEEE Trans. Biomed. Circuits Syst. 9 (5) (2015) 662-669. 